Ann. Biol. anim. Bioch. Biophys., 1977, 17 (5 B), 799-806.

\title{
Evolution, pendant la croissance, de la cellularité du tissu adipeux chez le lapin et l'agneav
}

\author{
par J. NOUGUĖS, A. VÉZINHET \\ avec la collaboration technique de Odette MOULIERAC, Thérèse CHERY et E. BOUTHIER \\ Station de Physiologie animale I.N.R.A., \\ E.N.S.A., Place Viala, 34600 Montpellier Cedex.
}

Summary. - Postnatal development of adipose tissue cellularity in the growing rabbit and lamb.

The evolution of adipose tissue cellularity has been studied in 1 to 20 -month old male New Zealand rabbits and in 10 to 250 -day old male Merinos d'Arles lambs.

Four adipose tissues located in the perirenal, peristomacal, interscapular and neck regions were studied in the rabbit. The increase in mean fat cell volume mostly occurs until the age of 6 months, when adipocyte size stabilizes in perirenal, peristomacal and interscapular fat pads. Subcutaneous neck adipocytes are smaller during the whole growth period studied (1 to 20 months). Growth of perirenal and interscapular fat pads until month 6 is due to an increase in both the number and size of adipocytes. However, between months 6 and 10, the weight of these fat pads still doubles; this is due to uninterrupted increase in the number of fat cells. Beyond the age of 10 months, the cellularity of these two fat pads is stable.

Four adipose tissues located in the perirenal, peristomacal, intermuscular and subcutaneous dorsal regions were studied in the lamb. Postnatal growth of intermuscular, peristomacal and subcutaneous adipose tissues until the age of 100 days is due to an increase both in the number and size of adipocytes; beyond this age, it can be attributed only to an increase in size. On the other hand, perirenal adipose tissue accumulation during the postnatal period is due almost entirely to an increase in adipocyte size. Contrary to that in the rabbit, the finishing growth of adipose tissue in the sheep is due to adipocyte hypertrophy.

In both species, the large variability in the weight of adipose tissue is generally related to a variation of the size and the number of fat cells.

\section{Introduction.}

L'évolution quantitative de la masse adipeuse, au cours de la croissance, est fonction de deux paramètres qui sont la taille et le nombre des cellules qui la constituent. La connaissance de l'évolution de ces deux paramètres est donc nécessaire à 
une meilleure compréhension du développement du tissu adipeux. D'autre part, cette connaissance est intéressante sur le plan physiologique et dans le cas des espèces animales domestiques sur le plan zootechnique. Elle permet, en effet, d'étudier la variation des activités métaboliques de ce tissu en relation avec l'évolution de la taille et du nombre des cellules (Vézinhet ef Nouguès, 1973) et de mieux définir les modalités de croissance et de changement des caractéristiques cellulaires des populations d'adipocytes de chaque tissu adipeux, dont la répartition ef la masse sont des facteurs importants d'appréciation de la qualité des carcasses d'animaux de boucherie (Prud'hon et al, 1970, Vézinhet et Prud'hon, 1975).

Les études relatives à l'évolution de la cellularité des différents tissus adipeux, au cours de leur développement, sont relativement récentes. La majorité de ces travaux a été réalisée chez le Rat ef la Souris (Hirsch et Han, 1969 ; Salans et al., 1972 ; Greenwood ef Hirsch, 1974 ; Hellman ef al, 1962 ; Lemonnier, 1972) et quelquesuns ont été réalisés chez l'Homme (Hirsch et Knittle, 1970 ; Sjöström et Björntorp, 1974), chez des porcins, des ovins et des bovins (Hood, 1972 ; Haugeback, 1973 ; Anderson et Kauffman, 1973 ; Vernon, 1976).

Ce travail rapporte les résultats concernant l'évolution de la cellularité de quatre tissus adipeux chez le Lapin et l'Agneau en croissance.

\section{Matériel et méthodes.}

Les animaux utilisés dans cette étude sont d'une part des lapins mâles Néozélandais âgés de 1 à 20 mois et d'autre part des agneaux mâles Mérinos d'Arles âgés de 10 à 250 jours. Les régimes alimentaires étaient dispensés ad libitum pour les individus de chaque espèce.

Quatre tissus adipeux de localisations anatomiques différentes ont été étudiés chez les deux espèces. Ce sont pour le Lapin les tissus périrénal, péristomacal, interscapulaire et sous-cutané du cou et pour l'Agneau les tissus périrénal, péristomacal (toilette), intermusculaire et sous-cutané dorsal. Dans le cas de cette dernière espèce, les échantillons de tissu adipeux étaient prélevés par biopsie.

La mesure de la taille des cellules de ces tissus était effectuée sur des adipocyłes isolés par traitement du tissu à la collagénase selon la technique de Rodbell (1964). Dans un travail précédent (Nouguès, 1975) nous avons montré que les adipocyles isolés par ce traitement constituaient un échantillon représentatif du tissu adipeux étudié. Des échantillons de 0,5 à $1 \mathrm{ml}$ de la suspension cellulaire étaient fixés par une solution à 2 p. 100 de tetroxyde d'osmium dans un tampon phosphate $(\mathrm{pH}=7,3)$. Le diamètre ef le volume des adipocytes fixés étaient mesurés en utilisant un appareil électronique (Coulter Counter, modèle ZB) muni d'un tube à orifice de 280 ou $400 \mu \mathrm{m}$ selon la nature du tissu adipeux étudié et l'âge des animaux, dont il provient.

L'estimation du nombre des cellules adipeuses, dans les divers tissus, a été réalisée par une méthode indirecte basée sur le rapport entre le poids en lipide du tissu adipeux ef le poids en lipide d'une cellule « moyenne » considérée comme représentative de chaque tissu étudié. Dans le cas de l'Agneau, où les tissus adipeux ont été prélevés par biopsie, les données relatives au poids de ces tissus ont été recueillilies au laboratoire après dissection des demi-carcasses d'un loł de 45 agneaux mâles 
Mérinos d'Arles répartis dans les six groupes d'âge étudiés (Vezinhet et Prud'hon, 1975). Les résultats présentés dans ce travail ne concernent que les cellules adipeuses différenciées, ou adipocytes, contenant une ou plusieurs inclusions lipidiques.

\section{Résultats et discussion.}

Dans la figure 1 sont représentées les évolutions du volume moyen des adipocytes de quatre tissus adipeux chez le Lapin. L'augmentation du volume moyen des adipocytes des tissus périrénal, péristomacal et interscapulaire est importante jusque vers l'âge de 105 jours, puis elle se poursuit encore faiblement jusqu'à 180 jours, où la taille des cellules tend à se stabiliser. Le tissu du cou présente, de 30 à 300 jours, une augmentation continue de l'ordre de 13 fois du volume moyen des adipocytes contre 4 à 6 fois pour les autres tissus. Il se distingue de ces derniers par le fait qu'il

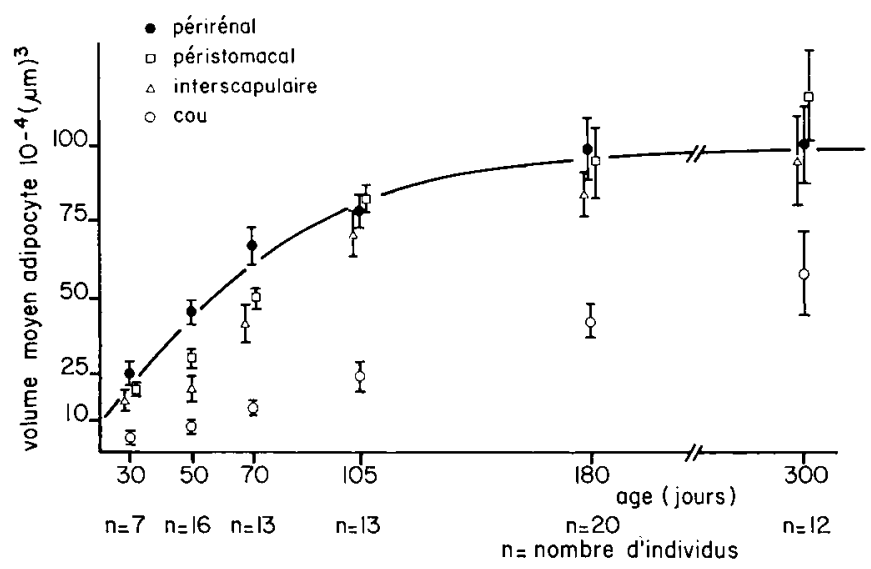

FIG. 1. - Evolution du volume des adipocyles de quatre tissus adipeux au cours de la croissonce du Lapin (moyenne ef erreur standard).

conserve, tout au long du développement du tissu adipeux, des cellules de plus petite taille. Les résultats obtenus au laboratoire (Vézinhet et Nouguès, 1973) sur l'activité métabolique, entre 30 et 180 jours, des dépôts étudiés montrent que les cellules des tissus adipeux superficiels, en particulier du cou, ont, jusque vers 150 jours, une activité lipolytique supérieure à celle des cellules des tissus adipeux profonds, pour une activité lipogénique équivalente à ces derniers. Ceci explique probablement le fait que les tissus adipeux superficiels présentent au début de leur développement des adipocytes d'une taille inférieure à celle des tissus adipeux profonds. Il faut aussi remarquer que les tissus du cou et interscapulaire sont, chez le Lapin, pendant la période périnatale, du type brun (Dawkins et Hull, 1964), spécialisés dans la production de chaleur et caractérisés, entre autres, par des cellules de plus petite taille ; ces dépôts se transforment progressivement, après la naissance, en tissu adipeux de type blanc (Derry et al., 1972). 
Chez le Lapin, l'évolution du nombre d'adipocytes (tabl. 1) a été étudiée dans les tissus périrénal et interscapulaire, qui peuvent être prélevés avec une grande précision à tous les âges. L'augmentation du poids des tissus, entre 30 et 70 jours, est due en grande partie à un accroissement du volume des adipocytes, leur nombre aug-

\section{TABLEAU 1}

Evolution du poids moyen ef du nombre d'adipocytes de deux tissus adipeux du Lapin (moyenne \pm erreur standard)

\begin{tabular}{cccccc}
\hline & & \multicolumn{2}{c}{ Périrénal } & \multicolumn{2}{c}{ Interscapulaire } \\
\cline { 3 - 6 } $\begin{array}{c}\text { Age } \\
(\mathrm{j})\end{array}$ & $\begin{array}{c}\text { Nombre } \\
\text { individus }\end{array}$ & $\begin{array}{c}\text { Poids frais } \\
(\mathrm{g})\end{array}$ & $\begin{array}{c}\text { Nombre adipocytes } \\
\times 10^{-6}\end{array}$ & $\begin{array}{c}\text { Poids frais } \\
(\mathrm{g})\end{array}$ & $\begin{array}{c}\text { Nombre } \\
\text { adipocytes } \\
\times 10^{-6}\end{array}$ \\
\hline 30 & 8 & $2,71 \pm 0,60$ & $7,177 \pm 0,608$ & $1,47 \pm 0,26$ & $7,429 \pm 0,685$ \\
\hline 50 & 10 & $3,64 \pm 0,50$ & $10,536 \pm 1,159$ & $1,89 \pm 0,23$ & $9,506 \pm 1,209$ \\
\hline 70 & 8 & $7,65 \pm 1,67$ & $11,342 \pm 2,063$ & $4,13 \pm 0,59$ & $9,224 \pm 0,507$ \\
\hline 105 & 13 & $15,39 \pm 1,98$ & $23,417 \pm 3,102$ & $7,28 \pm 0,53$ & $10,157 \pm 0,425$ \\
\hline 180 & 11 & $32,09 \pm 3,12$ & $42,133 \pm 4,633$ & $13,42 \pm 1,21$ & $16,312 \pm 0,739$ \\
\hline 300 & 6 & $88,76 \pm 14,76$ & $113,181 \pm 24,836$ & $20,35 \pm 3,55$ & $28,495 \pm 4,300$ \\
\hline 600 & 14 & $84,48 \pm 8,94$ & $97,690 \pm 8,930$ & $25,77 \pm 2,89$ & $32,170 \pm 3,461$ \\
\hline
\end{tabular}

mentant faiblement. Par contre, l'augmentation pondérale des dépôts entre 105 et 180 jours est surfout due à un accroissement du nombre des adipocytes, l'augmentation de la taille moyenne des cellules étant faible dans cette période. Entre les âges de 180 et 300 jours, il y a encore un accroissement pondéral très important des dépôts, plus du double dans le cas du tissu adipeux périrénal qui est dú à l'apparition de nouveaux adipocytes. Il n'y a pas dans cette période d'évolution de la taille moyenne des cellules qui puisse expliquer cet accroissement important du poids des tissus. Enfin, au-delà de 300 jours, la cellularité et le poids du tissu adipeux sont fixés, des animaux de 600 jours présentant des valeurs comparables à ceux d'animaux âgés de 300 jours.

Ainsi, dans le cas du tissu adipeux superficiel ou profond du Lapin, l'apparition de nouveaux adipocytes se fait sur une période plus longue que l'accroissement de la taille moyenne des cellules et elle rend compte de la croissance tardive des tissus adipeux.

Dans la figure 2 sont représentées les évolutions, entre 10 et 250 jours, du volume moyen des adipocytes de quatre tissus adipeux chez l'Agneau. Entre ces âges, il y a une augmentation importante du volume moyen des cellules des différents tissus ; les volumes à 10 jours n'atteignent que 10 à 15 p. 100 de leurs valeurs à 250 jours. 
A partir de 150 jours commencent à apparaître des différences entre les valeurs du volume des adipocytes des divers dépôts, avec jusqu'à 250 jours des cellules plus volumineuses dans les dépôts péristomacal et intermusculaire. Entre les âges de 50 et 100 jours, on note un net ralentissement de l'augmentation du volume des adipocytes. Les raisons de ce phénomène restent obscures, elles ne peuvent être attribuées à une diminution de l'activité lipogénique des cellules adipeuses, qui reste à peu près constante dans cette période (Vézinhet, 1976). Passé cet âge, il y a une reprise importante de l'accroissement du volume des adipocytes que l'on peut relier à une augmentation de l'activité lipogénique des cellules adipeuses (Vézinhet, 1976).

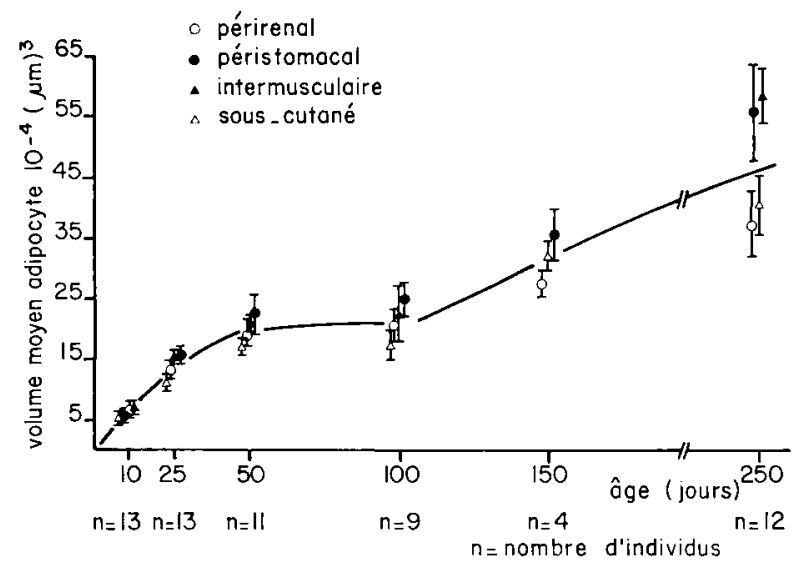

FIG. 2. - Evolution du volume moyen des adipocytes de quatre tissus adipeux de l'Agneau (moyenne et erreur standard).

Dans le tableau 2 sont regroupées les données moyennes concernant les évolutions du poids ef du nombre des adipocytes des quatre tissus étudiés. Le tissu périrénal se distingue des autres par le fait qu'il possède déjà à 10 jours environ 90 p. 100 du nombre total d'adipocytes présents dans ce dépôt à l'âge de 250 jours. L'augmentation pondérale de 4 fois du tissu dans cette période est surtout due à une hypertrophie des cellules. L'accroissement pondéral des tissus péristomacal, intermusculaire ef sous-cutané entre 10 et 100 jours est dô à la fois à une augmentation du volume et du nombre des adipocytes. Enfin, au-delà de 100 jours et jusqu'à 250 jours, dans le cadre de cette étude, l'accroissement pondéral de ces tissus qui est encore importante (voisine de 50 p. 100) est surout lié à une augmentation du volume moyen des adipocytes.

A l'inverse de ce qui se passe chez le Lapin, la croissance tardive du tissu adipeux chez le Mouton est due à une hypertrophie des adipocytes. Le tissu périrénal de l'Agneau présente un schéma de développement voisin du tissu adipeux épididymaire du rat, où la multiplication des adipocytes est limitée aux premières semaines de la vie alors qu'il y a une augmentation continue de la taille des cellules sur une longue durée (Hirsch et Han, 1969). Par ailleurs, les dépôts adipeux peuvent répondre différemment, selon leur localisation anatomique, à un même traitement. Par exemple, 
Lemonnier (1972) a montré que l'augmentation du poids des dépôts adipeux chez des souris rendues obèses par un régime hyperlipidique était due à une hyperplasie pour le dépôt périrénal, à une hypertrophie pour les dépôts épididymaire et sousculané et à une hyperplasie plus une hypertrophie pour le dépôt paramétrial.

TABLEAU 2

Evolution du poids moyen ( $上$ erreur standard) ef du nombre d'adipocytes de quatre tissus adipeux de l'Agneau

\begin{tabular}{|c|c|c|c|c|c|}
\hline & & Périrénal & Péristomacal & Intermusculaire & Sous-cutané \\
\hline $\begin{array}{l}\text { Age } \\
\text { (i) }\end{array}$ & $\begin{array}{l}\text { Nombre } \\
\text { individus }\end{array}$ & $\begin{array}{c}\text { Poids frais }(\mathrm{g}) \\
\text { Nombre } \\
\text { adipocytes } \\
\times 10^{-6}\end{array}$ & $\begin{array}{c}\text { Poids frais }(\mathrm{g}) \\
\text { Nombre } \\
\text { adipocytes } \\
\times 10^{-6}\end{array}$ & $\begin{array}{c}\text { Poids frais }(g) \\
\text { Nombre } \\
\text { adipocytes } \\
\times 10^{-6}\end{array}$ & $\begin{array}{c}\text { Poids frais (g) } \\
\text { Nombre } \\
\text { adipocytes } \\
\times 10^{-6}\end{array}$ \\
\hline 10 & 13 & $\begin{array}{l}61 \pm 7 \\
743,470\end{array}$ & $\begin{array}{c}33 \pm 7 \\
498,484\end{array}$ & $\begin{array}{l}75 \pm 20 \\
871,364\end{array}$ & $\begin{array}{l}64 \pm 10 \\
959,712\end{array}$ \\
\hline 25 & 13 & $\begin{array}{l}91 \pm 14 \\
610,153\end{array}$ & $\begin{array}{r}84 \pm 21 \\
468,354\end{array}$ & $\begin{array}{c}252 \pm 20 \\
1553,300\end{array}$ & $\begin{array}{l}252 \pm 38 \\
2171,202\end{array}$ \\
\hline 50 & 11 & $\begin{array}{r}133 \pm 23 \\
653,772\end{array}$ & $\begin{array}{r}165 \pm 22 \\
659,963\end{array}$ & $\begin{array}{c}421 \pm 61 \\
1890,762\end{array}$ & $\begin{array}{l}442 \pm 59 \\
2467,483\end{array}$ \\
\hline 100 & 9 & $\begin{array}{r}194 \pm 28 \\
932,688\end{array}$ & $\begin{array}{l}261 \pm 31 \\
1031,574\end{array}$ & $\begin{array}{l}620 \pm 74 \\
2672,806\end{array}$ & $\begin{array}{l}750 \pm 94 \\
3463,785\end{array}$ \\
\hline 150 & 4 & $\begin{array}{l}299 \pm 21 \\
1002,378\end{array}$ & $\begin{array}{l}588+65 \\
1225,070\end{array}$ & $861 \pm 64$ & $\begin{array}{c}1051 \pm 100 \\
3314,022\end{array}$ \\
\hline 250 & 12 & $\begin{array}{r}318 \pm 44 \\
881,406\end{array}$ & $\begin{array}{l}623 \pm 62 \\
1135,563\end{array}$ & $\begin{array}{c}1292 \pm 82 \\
2241,012\end{array}$ & $\begin{array}{c}1409 \pm 158 \\
3577,270\end{array}$ \\
\hline
\end{tabular}

Chez les deux espèces, on observe, à poids ou à âge égal, une variabilité très importante du poids des tissus adipeux avec des coefficients de variation atteignant 50 p. 100 . Elle est liée à une variation entre individus soit de la taille des cellules, soit de la taille et du nombre ou bien seulement du nombre. A titre d'exemple est présentée dans la figure 3 la variabilité de la taille des cellules et du poids de deux tissus adipeux chez le Lapin. Des individus ont des dépôts dont les poids sont très différents, mais qui présentent des histogrammes de distribution de la taille des cellules très voisins. L'écart dans le poids des tissus est, dans ce cas, attribuable uniquement à une différence importante du nombre des cellules.

A la fin de la croissance du tissu adipeux et lorsqu'il n'y a plus de formation en grand nombre de nouvelles cellules adipeuses on constate qu'il existe toujours une dispersion importante de la taille des cellules. Elle peut s'expliquer soit par la possibilité d'un renouvellement des cellules (Vodovar et al, 1971 ; Vodovar et al, 1972 ; Greenwood ef Hirsch, 1974), soit par des changements cycliques du métabolisme des adipocytes qui se traduiraient par des variations cycliques du volume des cellules. 
Différenis auteurs (Hartman ef al., 1971 ; Zinder et Shapiro, 1971 ; Di Girolamo et al., 1974) ont, en effet, observé chez le Rat, des modifications du métabolisme des adipocytes en rapport avec le changement de leur volume.

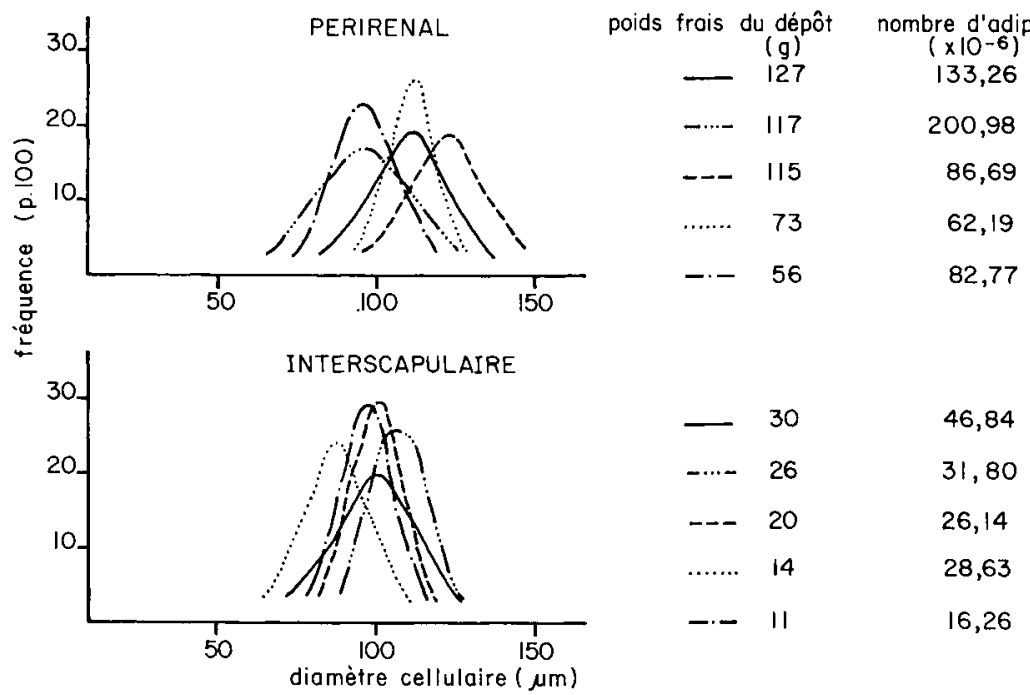

FIG. 3. - Variabilité de la faille des cellules (polygones de fréquence) ef du poids des dépôts adipeux périrénal et inferscapulaire chez cinq individus âgés de 300 jours.

\section{Conclusion}

Cette étude montre que les caractères du tissu adipeux et les modalités de son développement peuvent varier selon les espèces. Ainsi, en fin de croissance, le tissu adipeux du Lapin a des adipocytes plus volumineux que celui du Mouton. D'autre part, chez le Lapin, l'apparition de nouveaux adipocytes se fait sur une période plus longue que l'accroissement de la taille moyenne des cellules et elle rend compte de la croissance tardive des tissus adipeux. Le processus inverse est observé dans le cas du mouton. Enfin, le fait d'étudier des dépôts adipeux de différentes localisations anatomiques nous a montré qu'ils ne sont pas tous identiques dans leurs caractères et les modalités de leur développement. Par exemple, chez le Lapin, les adipocyłes du cou sont d'une taille moyenne bien inférieure à celle des autres dépôts et chez l'Agneau, au-delà de 150 jours les adipocytes des dépôts péristomacal et interscapulaire deviennent plus volumineux que ceux des autres dépôts. Par ailleurs, chez cette espèce, le dépôt périrénal est différent des autres par une faible augmentation postnatale du nombre des adipocytes, sa croissance étant en majeure partie due à une augmentation du volume de cellules déjà existantes.

Réunion Groupe Développement INRA/Productions animales Thiverval-Grignon, 14-15 avril 1976.

Remerciements. - Ce travail a été réalisé avec l'aide de la D.G.R.S.T., contrat 7371654 et de I'I.N.R.A., ATP « Tissu adipeux » nº 65532. 


\section{Références}

ANDERSON D. B., KAUFFMAN R. G., 1973. Cellular and enzymatic changes in porcine adipose tissue during growth. J. Lipid Res., 14, 160-168.

DAWKINS M. J. R., HULL D., 1964. Brown adipose tissue and the response of new-born rabbits to cold. J. Physiol., 172, 216-238.

DERRY D. M., MORROW E., SADRE N., FLATTERY K. V., 1972. Brown and white fat during the life of the rabbit. Develop. Biol., 27, 204-216.

Di GIROLAMO M., HOWE M. D., ESPOSITO J., THURMAN L., OWENS J. L., 1974. Metabolic patterns and insulin responsiveness of enlarging fat cells. J. Lipid Res., 15, 332-337.

GREENWOOD M. R. C., HIRSCH J., 1974. Postnatal development of adipocyte cellularity in the normal rat. J. Lipid Res., 15, 474-483.

HARTMAN A. D., COHEN A. I., RICHANE C., HSU T., 1971. Lipolytic response and adenyl cyclase activity of rat adipocytes as relafed to cell size. J. Lipid Res., 12, 498-505.

HAUGEBACK C., 1973. Effects of nutrition and age on adipose tissue growth and development in lambs. Thèse, Univ. Missouri, Columbia, pp. 166.

HIRSCH J., HAN P. W., 1969. Cellularity of rat adipose tissue : Effects of growth, starvation and obesity. J. Lipid Res., 10, 77-82.

HIRSCH J., KNITTLE J. L., 1970. Cellularity of obese and non obese human adipose tissue. Fed. Proc., 29, 1516-1521.

HELLMAN B. O., TÄLJEDAL I. B., WESTMAN S., 1962. Morphological characteristics of the epididymal adipose tissue in normal and obese hyperglycemic mice. Acia morphol. neerl. scand., 5, 182-189.

HOOD R. L., 1972. Adipose tissue cellularity and lipogenic activity in porcine and bovine animals. Thèse, Univ. Minnesota, pp. 298.

LEMONNIER D., 1972. Effect of age, sex and site on the cellularity of the adipose tissue in mice and rats rendered obese by a high fat diet. J. clin. Invest., 51, 2907-2915.

NOUGUÈS J., 1975. Adipocyte growth of four adipose deposits in rabbit. Ann. Biol. anim. Bioch. Biophys., 15, 541-546.

PRUD'HON M., VÉZINHET A., CANTIER J., 1970. Croissance, qualités bouchères et coût de la production des lapins de chair. Bull. tech. Inform., 248, 203-212.

SALANS L. B., ZARNOWSKI M. J., SEGAL R., 1972. Effect of insulin upon the cellular character of rat adipose tissue. J. Lipid Res., 13, 616-623.

SJÖSTRÖM L., BJÖRNTORP P., 1974. Body composition and adipose tissue cellularity in human obesify. Acta med. scand., 195, 201-211.

VERNON R. G., 1976. Perirenal adipose tissue development in the neonatal lamb. Biochem. Soc. Transac., 4, 228-229.

VÉZINHET A., NOUGUĖS J., 1973. In vitro lipolytic activity of porcine growth hormone in the rabbit : effects of location, number and size of adipocytes, 77-81. In VAGUE J., BOYER J. The regulation of the adipose tissue mass. Excerpta Medica, Amsterdam.

VÉZINHET A., PRUD'HON M., 1975. Evolution of various adipose deposits in growing rabbits and lambs. Anim. Prod., 20, 363-370.

VÉZINHET A., 1976. Etude du tissus adipeux chez l'agneau et le lapin : Développement, lipolyse, lipogenèse. Influence de l'hypophysectomie et de l'hormone de croissance. Thèse Etat, Univ. Montpellier, pp. 167.

VODOVAR N., DESNOYERS F., FRANÇOIS A., 1971. Origine et évolution des adipocytes mésentériques du porcelet avant la naissance. Aspect ultrastructural. J. Microsc., 11, 265-284.

VODOVAR N., DESNOYERS F., FLANZY J., 1972. Dépôt adipeux mésentérique du porcelet. Etude morphologique. Ann. Biol. anim. Bioch. Biophys., 12, 243-262.

ZINDER O., SHAPIRO B., 1971. Effect of cell size on epinephrine and ACTH induced fatty acid release from isolated fat cells. J. Lipid Res., 12, 91-95. 